\title{
Histone deacetylase inhibitors SAHA and sodium butyrate block G1-to-S cell cycle progression in neurosphere formation by adult subventricular cells
}

Qiong Zhou', Clifton L Dalgard', Christopher Wynder ${ }^{2}$ and Martin L Doughty ${ }^{1 *}$

\begin{abstract}
Background: Histone deacetylases (HDACs) are enzymes that modulate gene expression and cellular processes by deacetylating histones and non-histone proteins. While small molecule inhibitors of HDAC activity (HDACi) are used clinically in the treatment of cancer, pre-clinical treatment models suggest they also exert neuroprotective effects and stimulate neurogenesis in neuropathological conditions. However, the direct effects of HDACi on cell cycle progression and proliferation, two properties required for continued neurogenesis, have not been fully characterized in adult neural stem cells (NSCs). In this study, we examined the effects of two broad class I and class II HDACi on adult mouse NSCs, the hydroxamate-based HDACi suberoylanilide hydroxamic acid (vorinostat, SAHA) and the short chain fatty acid HDACi sodium butyrate.

Results: We show that both HDACi suppress the formation of neurospheres by adult mouse NSCs grown in proliferation culture conditions in vitro. DNA synthesis is significantly inhibited in adult mouse NSCs exposed to either SAHA or sodium butyrate and inhibition is associated with an arrest in the G1 phase of the cell cycle. HDACi exposure also resulted in transcriptional changes in adult mouse NSCs. Cdk inhibitor genes p21 and p27 transcript levels are increased and associated with elevated H3K9 acetylation levels at proximal promoter regions of p21 and p27. mRNA levels for notch effector Hes genes and Spry-box stem cell transcription factors are downregulated, whereas pro-neural transcription factors Neurog1 and Neurod1 are upregulated. Lastly, we show HDAC inhibition under proliferation culture conditions leads to long-term changes in cell fate in adult mouse NSCs induced to differentiate in vitro.

Conclusion: SAHA and sodium butyrate directly regulate cdk inhibitor transcription to control cell cycle progression in adult mouse NSCs. HDAC inhibition results in G1 arrest in adult mouse NSCs and transcriptional changes associated with activation of neuronal lineage commitment programs and a reduction of stem/progenitor state. Changes in differentiated cell state in adult mouse NSCs treated with HDACi under proliferation culture conditions suggests an intrinsic relationship between multipotency, cell cycle progression and HDAC activity in these cells.
\end{abstract}

Keywords: suberoylanilide hydroxamic acid, vorinostat, sodium butyrate, cyclin-dependant kinase inhibitor, p21 (Cip1/Waf1/Cdkn1a), p27 (Kip1/Cdkn1b), cell cycle, chromatin immunoprecipitation

\footnotetext{
*Correspondence: mdoughty@usuhs.mil

${ }^{1}$ Department of Anatomy, Physiology and Genetics, Center for Neuroscience and Regenerative Medicine, Uniformed Services University of the Health

Sciences, 4301 Jones Bridge Road, Bethesda, Maryland 20814, USA

Full list of author information is available at the end of the article
} 


\section{Background}

Adult neural stem cell (NSC) maintenance and differentiation is controlled by intrinsic and extrinsic factors. Many developmental cues have been shown to operate in the adult NSC niche including Wnt [1], sonic hedgehog [2,3], bone morphogenic protein [4] and notch signaling $[5,6]$. More recently the modification of histone proteins has been identified as an epigenetic regulator of adult neurogenesis [7-9]. Gene expression is epigenetically regulated by enzymatic modifications of histone proteins and changes in histone acetylation by the opposing activities of histone acetyltransferases (HATs) and histone deacetylases (HDACs) is considered the more dynamic form of regulation. HDACs catalyze the removal of an acetyl moiety from the $\varepsilon$-amino group of target lysine residues in histone proteins (reviewed in Grayson et al 2010) and histone deacetylation leads to a condensed chromatin structure that is primarily associated with the repression of transcription (it should be noted HDACs deacetylate other non-histone proteins such as $\alpha$-tubulin and $\beta$-catenin, see [10]).

The ability to inhibit HDAC activity with small molecule HDAC inhibitors (HDACi) has attracted considerable therapeutic attention. Initial interest focused on the application of HDACi as anti-cancer agents and suberoylanilide hydroxamic acid (vorinostat, SAHA, Zolinza) is the first HDACi approved by the FDA for cancer therapy. More recently, therapeutic interest in HDACi has broadened to non-malignant conditions effecting the nervous system [11]. Pre-clinical treatment models demonstrate HDACi exert neuroprotective effects and stimulate neurogenesis in traumatic brain injury (TBI) and ischemia $[12,13]$, restore learning and memory in TBI and neurodegenerative mice [14,15], enhance neuronal differentiation and synaptic plasticity $[16,17]$ and exert antidepressant-like effects [18]. However these same HDACi have also been reported to both prevent [19] or induce neuronal apoptosis in culture [20,21], a contradiction that is likely the result of differences in neuronal cell type, the culture conditions employed and the type of HDACi molecule tested.

In an effort to determine the cell specific effects of HDACi on adult neurogenesis, we have investigated the effects of the broad class I and class II HDAC inhibitors SAHA (a hydroxamate-based HDACi) and sodium butyrate (a short chain fatty acid) on adult mouse NSC biology in vitro. Our data indicate these two HDACi exert similar anti-proliferative effects in vitro by blocking G1to-S phase progression in adult mouse NSCs. G1 arrest is associated with the up-regulation of expression of cyclin-dependant kinase (cdk) inhibitors, the down-regulation of stem/progenitor transcription factors and upregulation of pro-neural transcription factors in adult
NSCs. Chromatin immunoprecipitation (ChIP) confirms HDACi directly regulate cdk inhibitor expression in adult mouse NSCs. Finally, we show HDACi treatment under proliferation culture conditions leads to longterm changes in cell fate in adult NSCs induced to differentiate in vitro. Combined these data indicate an intrinsic relationship between multipotency, cell cycle progression and HDAC activity in adult mouse NSCs.

\section{Results}

\section{SAHA and NaB inhibit neurosphere formation by adult mouse NSCs in vitro}

We have examined the effects of two HDAC inhibitors, the hydroxamate-based HDAC inhibitor suberoylanilide hydroxamic acid (vorinostat, SAHA) and the short chain fatty acid sodium butyrate $(\mathrm{NaB})$. SAHA and $\mathrm{NaB}$ are broad class I and class II HDAC inhibitors effective in micromolar $(\mu \mathrm{M})$ and millimolar $(\mathrm{mM})$ ranges respectively.

We examined the effects of $1 \mu \mathrm{M}$ SAHA and $1 \mathrm{mM}$ $\mathrm{NaB}$ on adult NSCs grown in proliferation culture conditions in vitro. Pharmacokinetics indicate $1 \mu \mathrm{M}$ SAHA treatment falls within the range of clinical use for this compound (effective anti-tumor oral doses of 200-400 mg vorinostat produce peak serum concentrations of 1.1-1.2 $\mu \mathrm{M}$, see [22]). Western blot analysis of whole cell extracts from adult NSCs treated with HDACi for 48 hours confirms SAHA and $\mathrm{NaB}$ treatment results in an increase in histone 3 (H3) acetylation levels when compared to DMSO and water vehicle controls (Figure 1a).

HDACi treatment was associated with clear differences in adult NSC behavior in culture. HDACi-treated adult NSCs exhibited a static behavior when grown in proliferative conditions compared to vehicle-treated controls and failed to produce neurospheres of any significant size or density in cultures (Figure 1b). Quantitative analysis of adult NSCs after 7-days HDACi treatment confirms SAHA and $\mathrm{NaB}$ treatment dramatically inhibits the formation of neurospheres (diameter $\geq$ $50 \mu \mathrm{m})$ and increase the prevalence of small ( $\geq 4$ cells, diameter $<50 \mu \mathrm{m}$ ) cell clusters in culture (Figure 1c). HDACi treatment resulted in minor cell death in culture that was most apparent during the first 1-2 days of treatment. However, quantitative analysis indicates these toxicity effects were not large-scale - LIVE/DEAD staining and flow cytometry reveals SAHA and $\mathrm{NaB}$ exposure results in a $10 \%$ reduction in cell viability compared to vehicle controls after 48 hours treatment (Figure 1d), a difference that is not statistically significant ( $\mathrm{NaB}$ vs. vehicle $\mathrm{p}=0.10$, SAHA vs. vehicle $\mathrm{p}=0.12$, $\mathrm{t}$-tests, $\mathrm{n}=$ $3)$. Rather, the increased prevalence of small cell clusters suggests HDACi treatment results in a modestly toxic 


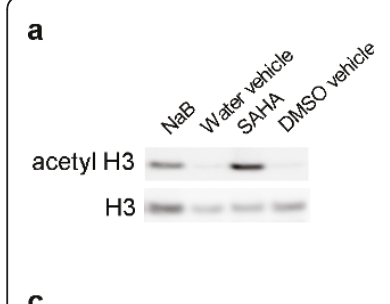

C

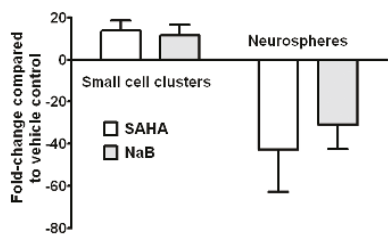

d
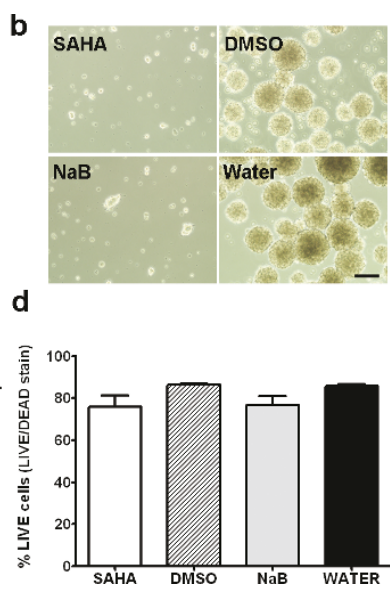

Figure $1 \mathrm{HDACi}$ treatment results in increased histone $\mathrm{H} 3$ acetylation, reduced neurosphere formation and minimal toxicity in adult mouse NSCs grown in proliferation conditions in culture. (a) Western blot of whole cell extracts demonstrates $\mathrm{SAHA}$ and $\mathrm{NaB}$ treatment result in increased levels of acetylated histone $\mathrm{H} 3$ compared to vehicle controls. (b) Light micrographs of HDACi/vehicle treated cultures illustrate the paucity of neurospheres in HDACi-treated cultures. (c) Quantitative analysis confirms SAHA and $\mathrm{NaB}$ inhibit the formation of neurospheres (diameter $\geq 50 \mu \mathrm{m}$ ) and increase the prevalence of small cell clusters ( $\geq 4$ cell, diameter $<50 \mu \mathrm{m})$. (d) Flow cytometry using LIVE/DEAD cell stain confirms SAHA and $\mathrm{NaB}$ are minimally toxic compared to vehicle controls ( $\mathrm{n}$ $=3$ ). Scale bar $=100 \mu \mathrm{m}$.

inhibition of the normal proliferative behavior of adult NSCs in our proliferative culture conditions.

\section{SAHA and NaB block G1- to-S cell cycle progression in adult mouse NSCs in vitro}

We quantified the effects of HDACi treatment on adult mouse NSC proliferation in vitro by comparing the levels of EdU (5-ethynyl-2'-deoxyuridine) incorporation in proliferation culture conditions. EdU is a nucleoside analog to thymidine and is incorporated into DNA during active DNA synthesis. Adult NSCs treated with SAHA or $\mathrm{NaB}$ were exposed to EdU $(10 \mu \mathrm{M})$ overnight (16 hours) following 48 hours HDACi treatment. Cell viability was measured by LIVE/DEAD stain and EdU incorporation compared in live cell gated populations. Flow cytometry measurement of EdU incorporation rates confirmed the addition of either SAHA or $\mathrm{NaB}$ significantly inhibited DNA synthesis in adult mouse NSCs in vitro (Figure 2a). SAHA treatment resulted in a 6.61 -fold reduction $(8.9 \pm 1.2 \%$ versus DMSO vehicle $56.3 \pm 7.0 \%, \mathrm{p}<0.01)$ and $\mathrm{NaB}$ a 5.26 -fold reduction $(10.4 \pm 5.2 \%$ versus water vehicle $54.5 \pm 7.5 \%, \mathrm{p}<$ $0.001)$ in $\mathrm{EdU}$ incorporation rates compared to vehicle controls.

Flow cytometry measurement of relative DNA content in combination with EdU incorporation was used to
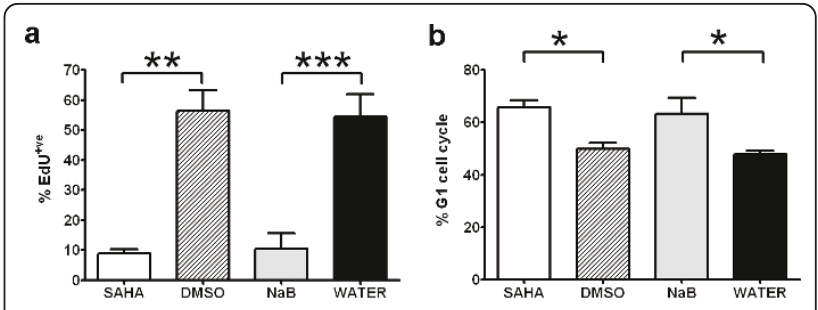

c

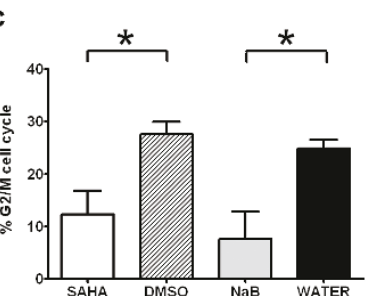

d

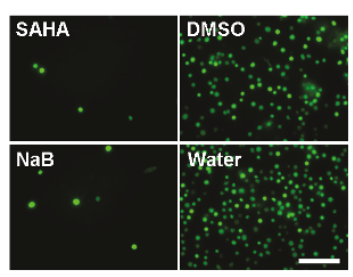

Figure 2 SAHA and NaB inhibit adult mouse NSC proliferation in culture and arrest cells in G1 phase of the cell cycle. (a) EdU flow cytometry of live cell-gated adult mouse NSCs demonstrates SAHA $\left({ }^{* *} \mathrm{p}<0.01\right)$ and $\mathrm{NaB}\left({ }^{* *} \mathrm{p}<0.001\right)$ significantly inhibit DNA synthesis. (b) Analysis of DNA content by flow cytometry reveals significant accumulation of adult mouse NSCs in G1phase of the cell cycle following SAHA $\left({ }^{*} p<0.05\right)$ and $\mathrm{NaB}\left({ }^{*} \mathrm{p}<0.05\right)$ treatment. (c) Correspondingly the proportion of cells in G2/M phase is significantly reduced following SAHA (*p $<0.05)$ and $\mathrm{NaB}\left({ }^{*} \mathrm{p}<\right.$ 0.05) treatment. (d) Fluorescence micrographs of HDACi/vehicle treated adult NSCs pulse-labeled with EdU for flow cytometry. Scale bar $=50 \mu \mathrm{m}$. Statistical comparisons performed using one-way ANOVA with post hoc Newman-Keuls multiple comparison tests. estimate the effects of SAHA and $\mathrm{NaB}$ on cell cycle progression in live cells. We used the Dean-Jett-Fox model (Flowjo software) to estimate percentage cell populations in G1 phase and G2/M phase of the cell cycle. This analysis revealed equivalent cell cycle effects in NSCs treated with SAHA or $\mathrm{NaB}$. SAHA or $\mathrm{NaB}$ treatment significantly increased the percentage of cells in G1 phase of the cell cycle (SAHA $65.6 \pm 2.8 \%$ versus $49.9 \pm$ $2.2 \%$ DMSO, $\mathrm{p}<0.05$ and $\mathrm{NaB} 63.3 \pm 6.0 \%$ versus 47.5 $\pm 1.3 \%$ water vehicle, $\mathrm{p}<0.05$ ), as shown in Figure $2 \mathrm{~b}$. Correspondingly, as shown in Figure 2c, SAHA or $\mathrm{NaB}$ treatment significantly decreased the percentage of cells in $\mathrm{G} 2 / \mathrm{M}$ phase (SAHA $12.3 \pm 4.5 \%$ versus $27.6 \pm 2.4 \%$ DMSO, $\mathrm{p}<0.05$ and $\mathrm{NaB} 7.7 \pm 5.2 \%$ versus $24.8 \pm 1.7 \%$ water vehicle, $\mathrm{p}<0.05)$. Combined these flow cytometry data indicate that the suppression of NSC proliferation by SAHA and $\mathrm{NaB}$ results in a G1-to-S phase block in cell cycle progression.

\section{Gene expression changes induced by SAHA and NaB} treatments vary in fold change but not directionality Genome-wide expression screening indicates HDAC inhibition is associated with expression changes in $\approx 2$ $5 \%$ of the genome [23]. We measured mRNA levels of a cohort of 18 genes implicated in cell cycle progression, stem cell maintenance and NSC fate using qRT-PCR. 
Table 1 Gene expression changes induced by SAHA or NaB treatment vary in fold change but not (+/-) directionality and are consistent with $\mathbf{G} 1$ arrest, suppression of stem/progenitor and activation of neuronal lineage commitment programs

\begin{tabular}{|c|c|c|c|}
\hline \multicolumn{4}{|c|}{ Genes Upregulated compared to vehicle control } \\
\hline Gene Symbol & Name & SAHA & $\underline{\mathrm{NaB}}$ \\
\hline Ctnnb1 & $\overline{\text { Catenin, beta-1 }}$ & $\overline{1.31 \pm 0.02}$ & $\overline{1.82} \pm 0.34$ \\
\hline Gli1 & glioma-associated oncogene & $5.26 \pm 1.90$ & $4.19 \pm 2.77$ \\
\hline Neurod1 & Neurogenic differentiation 1 & $4.28 \pm 1.78$ & $1.45 \pm 0.60$ \\
\hline Neurog1 & Neurogenin 1 & $9.86 \pm 4.35$ & $2.79 \pm 1.38$ \\
\hline Tcf4 & Transcription factor 4 & $1.50 \pm 0.34$ & $1.09 \pm 0.18$ \\
\hline p21 (Cdkn1a) & Cyclin-dependant kinase inhibitor 1a & $5.48 \pm 2.98$ & $3.35 \pm 0.99$ \\
\hline p27 (Cdkn1b) & Cyclin-dependant kinase inhibitor $1 \mathrm{~b}$ & $2.70 \pm 0.64$ & $2.34 \pm 0.31$ \\
\hline p57 (Cdkn1c) & Cyclin-dependant kinase inhibitor $1 \mathrm{c}$ & $1.89 \pm 0.48$ & $3.33 \pm 1.17$ \\
\hline Shh & Sonic hedgehog & $41.57 \pm 6.98$ & $6.31 \pm 2.37$ \\
\hline \multicolumn{4}{|c|}{ Genes Downregulated compared to vehicle control } \\
\hline Gene Symbol & $\underline{\text { Name }}$ & $\underline{\mathrm{SAHA}}$ & $\underline{\mathrm{NaB}}$ \\
\hline$\overline{A s c l 1}$ & 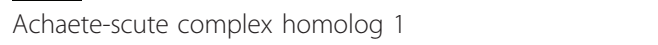 & $\overline{-2.36} \pm 0.71$ & $\overline{-3.43} \pm 0.92$ \\
\hline $\mathrm{Cdk} 2$ & Cyclin-dependant kinase 2 & $-4.64 \pm 1.91$ & $-8.37 \pm 5.87$ \\
\hline Cdk4 & Cyclin-dependant kinase 4 & $-3.35 \pm 0.70$ & $-4.18 \pm 0.17$ \\
\hline Cond1 & Cyclin D1 & $-1.55 \pm 0.63$ & $-1.87 \pm 0.17$ \\
\hline Hes1 & Hairy/enhancer of split, Drosophila homolog of, 1 & $-3.89 \pm 1.90$ & $-5.56 \pm 0.57$ \\
\hline Hes5 & Hairy/enhancer of split, Drosophila homolog of, 1 & $-11.87 \pm 1.18$ & $-23.85 \pm 3.15$ \\
\hline c-Myc & V-myc avian myelocytomatosis viral oncogene homolog & $-1.92 \pm 0.77$ & $-2.44 \pm 1.05$ \\
\hline Olig2 & Oligodendrocyte lineage transcription factor 2 & $-1.06 \pm 0.03$ & $-2.83 \pm 1.71$ \\
\hline Pax6 & Paired box gene 6 & $-2.04 \pm 1.18$ & $-4.22 \pm 1.92$ \\
\hline Sox1 & SRY-box 1 & $-4.46 \pm 3.33$ & $-4.96 \pm 2.52$ \\
\hline Sox2 & SRY-box 2 & $-3.08 \pm 1.60$ & $-3.44 \pm 1.06$ \\
\hline Sox9 & SRY-box 9 & $-2.35 \pm 0.25$ & $-1.24 \pm 1.06$ \\
\hline
\end{tabular}

+/- fold-changes in gene expression in adult mouse NSCs treated with SAHA or NaB were calculated from vehicle controls from $\geq 3$ independent $\mathrm{qRT}$-PCR trials

We harvested RNA for analysis after 48 hours treatment of NSCs in proliferation culture conditions with HDACi or vehicle. Our analysis revealed widespread changes in gene expression following HDACi exposure (Table1). Eight out of 18 genes analyzed showed increased expression and 10 decreased expression when compared to vehicle control. SAHA and $\mathrm{NaB}$ treatment induced greater than 2 fold expression changes in a majority of the genes tested (in both a positive $(+)$ and negative (-) direction from vehicle controls). Noticeably, the directionality (+/-) fold change of gene expression changes was consistent between the two HDACi treatments when compared to vehicle controls. We speculate this reflects the similar treatment outcome, G1 arrest, of SAHA and $\mathrm{NaB}$ exposure on adult NSCs.

Gene expression changes induced by SAHA and NaB are consistent with G1 arrest, a reduction in stem/progenitor state and activation of neuronal lineage commitment programs

Gene expression changes in HDACi treated adult NSCs are consistent with the inhibition of G1-to-S phase cell cycle progression. SAHA and $\mathrm{NaB}$ treatment result in increased transcription of cyclin dependant kinase inhibitors p21, p27 and p57, and the down-regulation of cyclin dependant kinases Cdk2 and Cdk4 (Table 1). Progression through $\mathrm{G1}$ and $\mathrm{S}$ phase of the cell cycle is dependent on Cdk2 and Cdk4 and the activity of these proteins is inhibited by binding of Cdk inhibitors p21, p27 and p57. We also examined genes with functions associated with stem/progenitor or neuronal cell fate. Our analysis revealed SAHA and $\mathrm{NaB}$ treatment results in the down-regulation of transcription factors associated with the maintenance of a stem/progenitor cell state and up-regulation of pro-neural transcription factors (Table 1). The progenitor cell cycle regulator c-Myc and stem cell maintaining SRY-box factors are downregulated by SAHA and $\mathrm{NaB}$ treatment, as are Notch effector bHLH transcription factors Hes 1 and Hes5 (Table 1). In contrast, mRNA levels of pro-neural bHLH transcription factors reveal variable HDACi effects on neuronal lineage commitment genes. Neurog1 and Neurod1 are upregulated whereas Ascl1 is downregulated in adult NSCs treated with SAHA or $\mathrm{NaB}$ (Table 1). In summary, qRT-PCR expression data from our gene cohort are consistent with G1 arrest accompanied by a 
reduction of stem/progenitor state and activation of Neurog1/Neurod1 neuronal lineage commitment programs.

\section{Increased transcription of Cdk inhibitors is associated with increased histone acetylation at the proximal promoter in HDACi treated adult mouse NSCs}

Transcription of the cell cycle regulator p21 is directly regulated by $\mathrm{HDAC} 1$ and $\mathrm{HDAC} 2$ [24-27] and is re-activated by $\mathrm{HDAC}$ inhibitors in tumor cells [28]. To test whether HDAC inhibitor treatment increased histone acetylation levels at the proximal promoter of the $p 21$ gene $(C d k n 1 a)$ in adults NSCs, we performed chromatin immunoprecipitation (ChIP) using antibodies to acetylated lysine 9 on histone H3 (anti-H3acK9). qPCR analysis reveals SAHA induces a 7.2-fold increase and $\mathrm{NaB}$ a 3.1 -fold increase in $\mathrm{H} 3 \mathrm{~K} 9$ acetylation levels at the $p 21$ promoter compared to DMSO and water vehicle controls respectively (Figure 3a). Statistical analysis confirms a significant increase in H3K9 acetylation in adult NSCs in response to SAHA $(\mathrm{p}<0.01)$ and $\mathrm{NaB}(\mathrm{p}<0.05)$, as well as significant interaction between the immunoprecipitation (IP) antisera $(\mathrm{p}<0.0001)$ and treatment $(\mathrm{p}<$ $0.05)$. We also analyzed H3K9 acetylation levels at the proximal promoter of $p 27(C d k n 1 b)$. Expression of p27 is increased in some but not all tumor cells by HDACi and is upregulated in human mesenchymal stem cells by SAHA treatment [29]. As shown in figure 3b, ChIP revealed a 2-fold increase $\mathrm{H} 3 \mathrm{~K} 9$ acetylation levels at the p27 promoter in response to SAHA treatment that was statistically significant $(\mathrm{p}<0.05)$. In contrast, no increase in $\mathrm{H} 3 \mathrm{~K} 9$ acetylation was observed at the $p 27$ promoter in response to $\mathrm{NaB}$ treatment (Figure $3 \mathrm{~b}$ ). Combined these data indicate transcriptional activation of p21 and p27 by SAHA is associated with increased H3K9 acetylation at proximal promoter regions, suggesting direct activation of these gene targets. Increased p21 expression and increased in H3K9 acetylation at the $p 21$ promoter in $\mathrm{NaB}$ treated cells suggests $\mathrm{NaB}$ similarly directly activates p21 transcription in adult NSCs. In contrast, the absence of significant changes in H3K9 acetylation at the $p 27$ promoter suggests the involvement of alternative mechanisms, possibly acetylation of non-histone proteins, in NaB-mediated increases in p27 expression in adult NSCs.

Treatment of proliferating adult mouse NSCs with HDACi leads to changes in differentiated cell fate

Inhibition of histone deacetylases promotes the acquisition of neuronal and suppresses glial cell fates in differentiating adult NSCs $[17,30]$. Based on these observations, we predicted HDACi treatment of proliferating adult mouse NSCs would lead to changes in cell fate in adult NSCs induced to differentiate in culture.

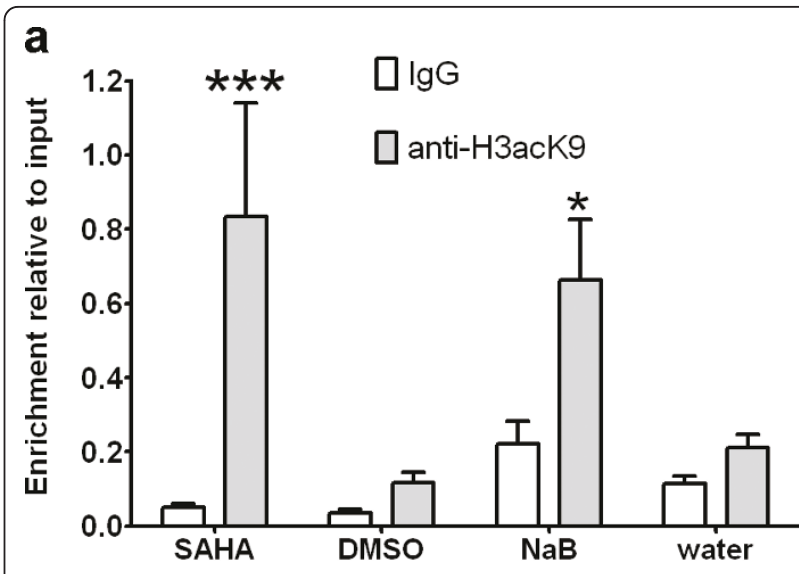

b

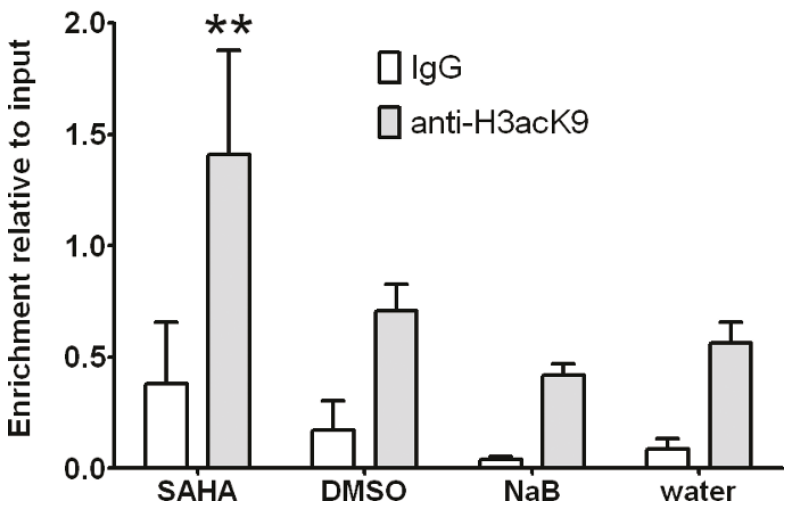

Figure 3 Upregulated p21 and p27 expression in adult mouse NSCs treated with HDACi is associated with increased acetylation at the proximal promoter. (a) Chromatin immunoprecipitation (ChIP) reveals significant increases in histone H3 lysine 9 acetylation levels at the proximal promoter of $p 21$ in SAHA $\left({ }^{* *} p<0.001\right)$ and NaB $\left({ }^{*} p<0.05\right)$ treated adult mouse NSCs. (b) ChIP reveals significant increases in histone $\mathrm{H} 3$ lysine 9 acetylation levels at the proximal promoter of $p 27$ in SAHA $\left(^{* *} p<\right.$ 0.01 ) but not NaB treated adult mouse NSCs. Statistical comparisons performed using two-way ANOVA with Bonferroni post-tests.

To test this hypothesis, we developed a 96-well plate immunofluorescence cell fate assay using antibodies to GFAP, Olig2 and NeuN as general markers of astrocytic, oligodendrocyte and neuronal cell fates. Adult NSCs were first treated with HDACi for 48 hours under proliferation culture conditions and then cultured for 14 days under differentiation conditions without HDACi. These assays revealed SAHA significantly reduced glial $(\mathrm{p}<0.001)$ and oligodendrocyte $(\mathrm{p}<0.05)$ differentiated cell fate in culture when compared to DMSO vehicle controls (Figure 4a). However NeuN immunoassays indicate this did not lead to a compensatory increase in neuronal cell fate (Figure 4a). Although $\mathrm{NaB}$ treatment similarly reduces the expression of glial and oligodendrocyte cell markers, these differences were not 


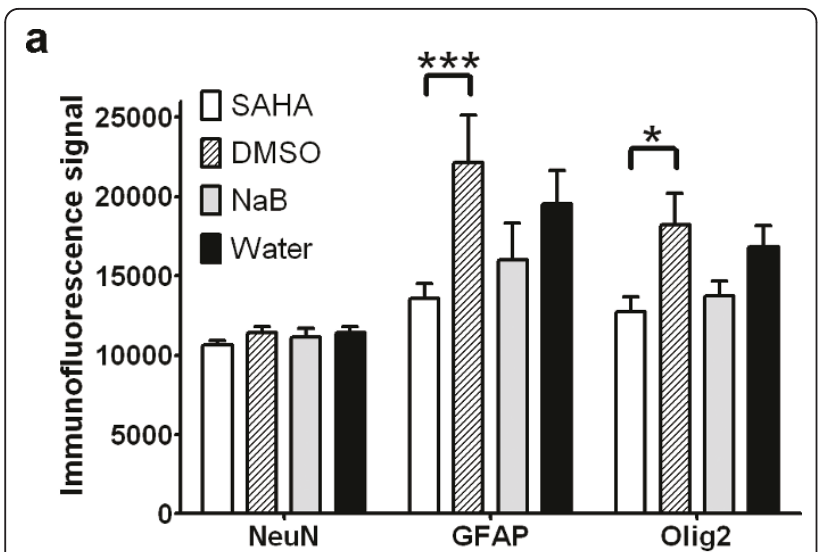

b

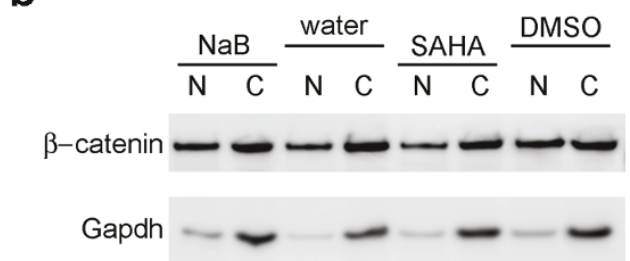

Laminin A/C

C

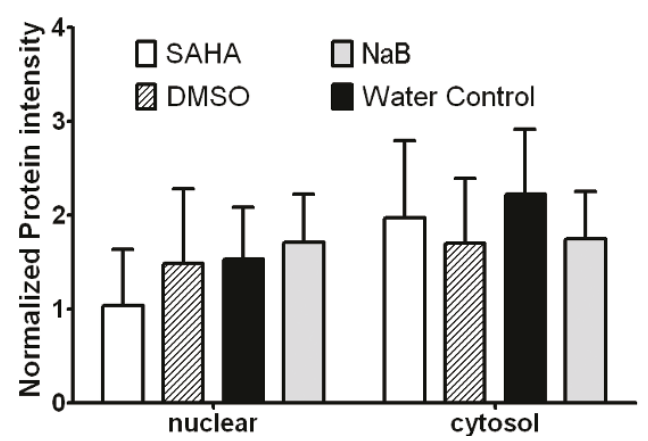

Figure 4 HDACi treatment under proliferation culture conditions leads to long-term changes in differentiated cell fate. (a) Immunofluorescence labeling of NeuN, GFAP and Olig2 cell fate markers reveals reduced expression of GFAP $\left({ }^{*} p<0.001\right)$ and Olig2 $\left.{ }^{*} p<0.05\right)$ in adult mouse NSCs treated with SAHA for 48 hours under proliferation culture conditions and then induced to differentiate for 14 days in culture (two-way ANOVA with Bonferroni post-tests). (b) Western blot of $\beta$-catenin protein in nuclear and cytoplasmic fractions of adult mouse NSCs treated with HDACi/ vehicle control under proliferation culture conditions. Gapdh and lamin A/C blots are included as loading controls. (c) SAHA and NaB do not significantly alter nuclear/cytoplasmic levels of $\beta$-catenin in adult mouse NSCs. Histogram of $\beta$-catenin Western blot signal intensities normalized to loading controls $(n=4)$.

significant compared to vehicle controls. As was the case with SAHA, $\mathrm{NaB}$ treatment did not alter NeuN expression levels (Figure 4a).

In an effort to identify molecular mechanisms responsible for SAHA-induced suppression of oligodendrocyte cell fate, we measured $\beta$-catenin protein levels in HDACi-treated adult NSCs by Western blot. Combined deletion of Hdac1 and Hdac2 in mice is reported to inhibit oligodendrocyte differentiation through the stabilization and nuclear translocation of $\beta$-catenin, which in turn represses Olig2 expression [31]. Hence our rationale was that SAHA inhibits Hdac1/2 in proliferating adult NSCs leading to increased nuclear localization of $\beta$-catenin and longer-term suppression of oligodendrocyte fates. However, Western blots $(\mathrm{n}=4)$ of adult NSCs treated with HDACi under proliferation conditions do not reveal significant changes in $\beta$-catenin nuclear localization in treated cells (Figure $4 \mathrm{~b}$ and $4 \mathrm{c}$ ). Fold-changes of $\beta$-catenin nuclear protein levels normalized to laminin $\mathrm{A} / \mathrm{C}$ reveal marginal opposing $+/$ - effects of SAHA $(-1.49 \pm 0.11)$ and $\mathrm{NaB}(1.36 \pm 0.33)$ treatment, indicating SAHA modulates cell fate via mechanisms independent of increased $\beta$-catenin stabilization.

\section{Discussion}

In this study we demonstrate the broad class I and class II histone deacetylase inhibitors SAHA and $\mathrm{NaB}$ block adult NSC proliferation in vitro by blocking G1-to-S progression. HDACi induced cell cycle blockade is accompanied by transcriptional changes consistent with G1 arrest, a reduction of stem/progenitor cell state and activation of neuronal lineage commitment programs in adult NSCs. Furthermore, HDACi treatment of adult NSCs in proliferation culture conditions leads to longerterm changes in cell fate when cells are induced to differentiate in culture.

\section{SAHA and NaB block G1-to-S cell cycle progression in} adult NSCs and activate cdk inhibitor expression

We have shown SAHA and $\mathrm{NaB}$ treatment inhibits adult NSC proliferation in vitro by arresting cells in G1 phase of the cell cycle. G1 arrest induced by SAHA or $\mathrm{NaB}$ treatment has been reported in fibroblasts [32], vascular smooth muscle cells [33] and numerous tumor cell types (see [28]). In many of these contexts, G1 arrest is associated with increased expression of p21 [34] indicating the anti-proliferative effects of SAHA and $\mathrm{NaB}$ are, in part, mediated by changes in the expression of cyclin dependant kinase inhibitors.

The functional link between HDAC-mediated regulation of cyclin dependant kinase inhibitor activity and cell cycle progression is supported by a number of genetic studies targeting HDAC genes in mice. Targeted deletion of Hdac1 in mice results in embryonic lethality associated with severe reductions in embryonic stem cell proliferation and increased p21 and p27 expression in null mutant embryos [27]. Furthermore, disruption of the $p 21$ gene $(C d k n 1 a)$ rescues the proliferation phenotype of Hdac1-/- mouse embryonic stem cells (but not 
the embryonic lethality of the mutation) and chromatin immunoprecipitations confirm the presence of HDAC1 at the $p 21$ promoter [26]. Similarly, combined deletion of $H D A C 1$ and $H D A C 2$ in primary fibroblasts and $\mathrm{B}$ cells results in a strong $\mathrm{G} 1$ cell cycle block that is accompanied by increased p 21 and p57 transcription [25], and loss of HDAC1/2 catalytic activity results in G1 arrest and up-regulation of p21 in primary and oncogenic-transformed embryonic fibroblasts [24]. Taken together these studies indicate HDAC1 and HDAC2 regulate G1-to-S cell cycle progression in multiple cell types by normally repressing the expression of cyclin dependant kinase inhibitor genes, in particular through transcriptional repression of $\mathrm{p} 21$.

In agreement with this, our data reveal SAHA and $\mathrm{NaB}$ upregulated p21 mRNA expression in adult mouse NSCs and that transcriptional activation is associated with increased $\mathrm{H} 3 \mathrm{~K} 9$ acetylation at the proximal promoter region of the p21 gene. This data indicates SAHA and $\mathrm{NaB}$ directly increases the acetylation of associated chromatin histone residues to upregulate p21 transcription in adult NSCs in vitro, a finding that implies class I and/or class II HDAC activity directly represses p21 gene transcription in adult NSCs to regulate cell proliferation. Similarly our data demonstrates SAHA and NaB upregulated p27 mRNA expression in adult NSCs. However, p27 transcriptional activation is associated with increased H3K9 acetylation at the gene's proximal promoter region of SAHA but not NaB treated adult NSCs. This suggests HDAC activity inhibited by SAHA but not $\mathrm{NaB}$ directly represses p27 transcription in adult NSCs to regulate cell proliferation. The fact that p27 mRNA levels are upregulated by $\mathrm{NaB}$ treatment irrespective of $\mathrm{H} 3 \mathrm{~K} 9$ acetylation changes suggests indirect $\mathrm{NaB}$ effects on p27 transcription in adult NSCs. We speculate that one of the various acetylated non-histone proteins such as p53 may provide the linkage between HDACi and p27 repression [34,35].

\section{SAHA and $\mathrm{NaB}$ treatment suppresses stem/progenitor and activates neuronal lineage commitment programs in adult NSCs}

Our expression data demonstrates SAHA and $\mathrm{NaB}$ treatment results in significant changes in gene transcription, changes that differed in magnitude but not directionality from vehicle controls reflecting the similar treatment outcomes of the two HDACi. The three signaling pathways represented within our gene cohort, Sonic Hedgehog [2,36], Wnt/ $\beta$-catenin [1] and Notch [37] signaling are known to combine to regulate NSC proliferation and neurogenesis in adult rodents. Thus the widespread changes in gene expression indicate HDAC inhibitors alter the output of each of the major regulatory pathways identified in adult NSCs. And since these pathways also control subsequent cell fate selection, it was not surprising that we observed alterations in cell markers such as Olig 2 when cells were induced to differentiate.

Overall, the expression data demonstrated HDACi treatment downregulates transcription factors implicated in the maintenance of stem/progenitor cell states and upregulates transcription factors that drive neuronal lineage commitment and differentiation. However, there are a few exceptions that merit discussion. These are the downregulation of pro-neural bHLH factor Ascl1 and the upregulation of Shh and Gli1 mRNAs. Ascl1 downregulation could result indirectly through the modulation of upstream activators such as Tlx [38]. ChIP data suggests direct HDACi effects at the Shh loci account for the increase in transcription of Shh and its target Gli1 (data not shown). The fact that Shh-Gli1 upregulation is accompanied by G1 arrest in our study suggests the mitogenic effects of Shh in adult NSCs [3] require normal HDAC function.

In addition to activating cell lineage commitment programs [28,39], HDACi also promote the re-setting of multipotency in terminally differentiated cell lineages by increasing induced pluripotent stem cell (iPS) re-programming efficiency [40-42]. Somatic cell re-programming reactivates expression of Nanog, a homeobox transcription factor necessary for maintaining multipotency in embryonic stem (ES) cells [43]. In ES cells, mSin3A-HDAC complex at the Nanog promoter acts to positively regulate Nanog expression under proliferating conditions and HDAC inhibition by TSA downregulates Nanog expression [44], a finding that is analogous to $\mathrm{SAHA}$ and $\mathrm{NaB}$ repression of multipotent factors in our adult mouse NSC cultures.

In our study both SAHA and $\mathrm{NaB}$ downregulate the expression of the stem cell maintaining factor Sox 2 in adult mouse NSCs. Conditional gene deletion in mice reveals Sox 2 is negatively regulated by Hdac 2 in adult neuroblasts and that Sox2 repression is necessary for adult neurogenesis - Hdac2-/- neuroblasts ectopically maintain Sox 2 expression, fail to mature into neurons and ultimately die of apoptosis [8]. Taking our data into consideration, HDAC regulation of Sox 2 expression in adult NSC lineage progression appears to be biphasic: in the first phase, class I and/or class II HDAC activity is required to maintain Sox 2 expression and the maintenance of stem/progenitor programs in self-renewing NSCs; in the second phase, HDAC2 functions to downregulate Sox 2 in neuroblasts and permit the full activation of neuronal differentiation programs.

\section{SAHA treatment leads to cell fate changes in differentiated adult NSCs}

Our cell fate analysis revealed SAHA treatment of adult NSCs under proliferation culture conditions lead 
to the long-term suppression of glial and oligodendrocyte cell fate markers GFAP and Olig2 in cells induced to differentiate. Using Olig1-Cre and floxed Hdac1 and Hdac2 mice, Ye et al. (2009) showed combined deletion of Hdac1 and Hdac2 in oligodendrocyte progenitor cells inhibited oligodendrocyte differentiation by repressing Olig2 expression. This effect was mediated by the stabilization (reduced phosphorylation) and nuclear translocation of $\beta$-catenin, which in turn negatively regulates oligodendrocyte development by repressing Olig2 expression [31]. These authors identified the transcription factor TCF7L2/TCF4 as a coeffector with $\beta$-catenin in the regulation of oligodendrocyte differentiation and speculate HDAC1/2 competition with $\beta$-catenin for TCF7L2 interaction converts TCF7L2 from a repressor to an activator of oligodendrocyte differentiation. Accordingly, we hypothesized HDACi induced increases in $\beta$-catenin stabilization and nuclear localization accounted for Olig2 suppression in our cell fate assays. However Western blot analysis failed to detect significant differences in nuclear protein levels of $\beta$-catenin in adult mouse NSCs treated with HDACi under proliferation culture conditions. We speculate HDACi effects on other targets offset this competitive interaction. One possible candidate is Hdac6, a class IIb HDAC. Hdac6 deacetylates $\beta$-catenin at lysine 49 (Lys49) to reduce $\beta$-catenin phosphorylation and promote $\beta$-catenin nuclear localization and c-myc induction [45]. Thus inhibition of Hdac1/2 and Hdac6 activity has the capacity to promote opposing effects on $\beta$-catenin stability and nuclear localization by increasing stability (and reducing phosphorylation) via inhibition of Hdac1/2 as well as decreasing stability and nuclear localization as a result of increased Lys49 acetylation and phosphorylation. Indeed differential sensitivities of Hdac1/2 and Hdac6 to SAHA and NaB inhibition might underlie the different $+/$ - fold-changes in $\beta$-catenin nuclear localization compared to vehicle controls). The fact that the effects of HDACi are consistent with anti-proliferative responses to pharmacological and genetic interventions targeting the canonical Wnt/ $\beta$-catenin signaling pathway in adult NSCs $[1,46]$ suggests the net effect of these molecules is to inhibit rather than activate this signaling pathway.

\section{Conclusion}

In summary, the broad class I and class II HDAC inhibitors SAHA and NaB blocked G1-to-S phase progression in proliferating adult NSCs in vitro. Gene expression changes induced by SAHA and $\mathrm{NaB}$ treatment in adult NSCs vary in fold change but not directionality, consistent with the comparable treatment outcomes of G1 arrest. In addition, the +/- direction of gene changes induced by SAHA and $\mathrm{NaB}$ treatment is consistent with G1 arrest accompanied by a reduction of stem/progenitor state and activation of neuronal lineage commitment programs. SAHA and $\mathrm{NaB}$ treatment induces increases in the transcription of $\mathrm{Cdk}$ inhibitors p21 and p27 in adult NSCs which was associated with elevated H3K9 acetylation levels at proximal promoter regions. This association is consistent with direct SAHA and $\mathrm{NaB}$ effects on cell cycle arrest genes in adult NSCs, in common with widely reported HDACi induced growth arrest in normal and transformed cells $[28,47]$. Finally, we show HDACi treatment under proliferation culture conditions leads to long-term changes in cell fate in adult NSCs induced to differentiate in vitro.

\section{Methods}

\section{Animals}

8-10 week old male and female (equal numbers) C57BL/ 6J mice were purchased from The Jackson Laboratory and housed in the Uniformed Services University's Center for Laboratory Animal Medicine prior to experimental use. Animals were handled in accordance with procedures approved by the Uniformed Services University of the Health Sciences Institutional Animal Care and Use Committee (IACUC). All research complied with DoD regulations as published in DoD Directive 3216.1. The University's Center for Laboratory Animal Medicine is a fully accredited institution with the Association for Assessment and Accreditation of Laboratory Animal Care (AAALAC).

\section{Neural stem cell culture}

Adult neural stem cells (NSCs) were harvested from the subventricular zone (SVZ) of adult C57BL/6J mice for culture. Mice were deeply anesthetized with vaporized isoflurane, decapitated, the whole brain dissected out and the forebrain cut in serial, coronal sections 1 mm thick using a Sorvall Tissue Chopper. The SVZ was dissected out from coronal sections and dissociated to a single cell suspension using Neural Tissue Dissociation kit (Mitenyi Biotech) according to the manufacturer's protocol. Cells were seeded at a cell density of $1 \times 10^{5}$ cells $/ \mathrm{ml}$ in mouse NeuroCult ${ }^{\circledR}$ NSC Basal Medium supplemented with mouse NeuroCult ${ }^{\circledR}$ NSC Proliferation Supplement, $20 \mathrm{ng} / \mathrm{ml} \mathrm{rh}$ EGF, 10 $\mathrm{ng} / \mathrm{ml}$ of $\mathrm{rh} \mathrm{FGF-b}$ and $2 \mathrm{ug} / \mathrm{ml}$ Heparin (all StemCell Technologies, Vancouver, BC, Canada). Cells were cultured at $37^{\circ} \mathrm{C}, 5 \% \mathrm{CO}_{2}$ and cultures passaged every 5-7 days. Cells were passaged a minimum of 5 times prior to experimental analysis to ensure a high enrichment of multipotent stem cells. Cells were dissociated to a single cell suspension in $0.025 \%$ Trypsin-EDTA (Invitrogen, Carlsbad, CA, USA) and re-seeded in culture medium at $1 \times 10^{5}$ cells $/ \mathrm{ml}$. 


\section{HDAC inhibitor Treatment}

HDAC inhibitors (HDACi) suberoylanilide hydroxamic acid (SAHA, Selleck chemicals, Houston, TX, USA) or sodium butyrate ( $\mathrm{NaB}$, Sigma, St. Louis, MO, USA) were added to NSC cultures 2 hours post-passage. SAHA or $\mathrm{NaB}$ were added to a final concentration in culture media of $1 \mu \mathrm{M}$ or $1 \mathrm{mM}$ respectively from freshly prepared solutions of $100 \mu \mathrm{M}$ SAHA/ DMSO and $100 \mathrm{mM} \mathrm{NaB} /$ water. An equal volume of DMSO or water was added to cultures as vehicle controls.

\section{Small cell cluster and neurosphere counts}

Following 7 days HDACi treatment, adult NSCs were incubated with a $0.2 \%$ solution of trypan-blue dye and small cell clusters/neurospheres excluding dye counted. Average small cell clusters/neurospheres were estimated from 10 randomly selected $2.1025 \mathrm{~mm}^{2}$ counting areas using an optical graticule and Zeiss inverted A1 microscope. The criteria for inclusion as small cell clusters were clusters of $\geq 4$ cells but a cell aggregate sphere diameter of $<50 \mu \mathrm{m}$. The criteria for neurospheres was a cell aggregate sphere of $\geq 50 \mu \mathrm{m}$ in diameter. Small cell cluster/neurosphere numbers were compared to values obtained from simultaneous vehicle control cultures to calculate fold-changes. Counting area selection and cell counting were independently performed by two "blinded" investigators.

\section{Flow Cytometry, Click-iTrM 5-ethynyl-2'-deoxyuridine (EdU) and CellCycle DNA labeling}

All procedures were performed according to the manufacturer's instructions (Invitrogen). Adult NSCs cultures were pulsed with $10 \mu \mathrm{M}$ 5-ethynyl-2'-deoxyuridine $(\mathrm{EdU})$ in cell culture media for 4 or 16 hours at $37^{\circ} \mathrm{C}$, $5 \% \mathrm{CO}_{2}$, the cells harvested and labeled with Alexa Fluor $^{\circledR} 488$ dye by Click-iT ${ }^{\mathrm{TM}}$ chemistry. For combined cell cycle analysis, cells were co-labeled with CellCycle 488-red (7-AAD) and LIVE/DEAD ${ }^{\circledR}$ Fixable Violet stains. For cells quantified for EdU content only, cells were co-labeled with LIVE/DEAD ${ }^{\circledR}$ Fixable Red stain (Invitrogen).

DNA content and proliferation status data acquisition was performed on a LRS II Flow Cytometer System at low flowrate with standard compensation and double discrimination parameters. Acquired data was analyzed using FlowJo 9.0.1 (Ashland, OR, USA). Briefly, events were first gated based on FSC and SSC parameters for the prominent cell population, then gated based on LIVE/DEAD signal. Live cells were analyzed for EdU incorporation for proliferation and also DNA content. Cell cycle analysis of the live population was performed in FlowJo using a Dean-Jeff-Fox model to compute the percentage of events in each phase.

\section{Real-time quantitative reverse transcription PCR (qRT-} PCR)

First-strand cDNA was synthesized from $1 \mathrm{ug}$ of total RNA using random primer/oligo(dT) primer according to the manufacturer's instructions (ABI, Carlsbad, CA, USA). Synthesized cDNA was diluted to final concentration of $10 \mathrm{ng} / \mathrm{ul}$ for qPCR using SYBR Green Master Mix (ABI) on an ABI 7500 Real-Time PCR System. Optimum primers were designed using NCBI's Primer BLAST http://www.ncbi.nlm.nih.gov/tools/primer-blast/. Primer sequence pairs used are listed in table 2. Primer specificity was confirmed by verifying a single PCR product had been generated using UV gel electrophoresis, as well as by confirming the melting temperature of the product had a single value on dissociation plots. Gene of interest (GOI) Ct values were normalized to internal control 18S, Actb and/or Hprt1 to calculate $\Delta \mathrm{Ct}$. Foldchanges (F) in gene of interest (GOI) expression were estimated using the $\Delta \Delta \mathrm{Ct}$ method: $\mathrm{F}=2^{-\Delta \Delta \mathrm{Ct}}$ where $\Delta \Delta \mathrm{C}_{\mathrm{t}}=\mathrm{GOI} \Delta \mathrm{C}_{\mathrm{t}}$ HDACi - GOI $\Delta \mathrm{C}_{\mathrm{t}}$ vehicle control. $\mathrm{Ct}$ values were measured in triplicate and recorded if the standard deviation was $<0.3$. All values represent a minimum of 3 biological replicates.

\section{Chromatin Immunoprecipitation (ChIP)}

Adult NSCs were treated for 10 minutes with $1 \%$ formaldehyde in PBS at room temperature. The cells were lysed in cell lysis buffer (5 mM PIPES ( $\mathrm{pH}=8.0$ ), 85 $\mathrm{mM} \mathrm{KCl}, 0.5 \% \mathrm{NP}-40$ and $1 \times$ protease Inhibitors, Pierce, Rockford, IL, USA) and the crude cell lysate transferred to a sonication buffer ( $1 \times$ PBS, $1 \%$ NP-40, $0.5 \%$ sodium deoxycholate and $1 \times$ protease Inhibitors, Pierce). The lysate was sonicated under conditions yielding fragments ranging from 500 to $1,000 \mathrm{bp}$. Samples were subsequently precleared at $4^{\circ} \mathrm{C}$ with recombinant protein $\mathrm{G}$ agarose beads (Roche, Indianapolis, IN, USA). Precleared lysate $(100 \mu \mathrm{l})$ diluted in immunoprecipitation buffer (0.01\% SDS/1.1\% Triton X-100/1.2 mM EDTA/16.7 mM Tris. $\mathrm{HCl}, \mathrm{pH} 8.0 / 167 \mathrm{mM} \mathrm{NaCl}$ ) was used for a 16 hour overnight immunoprecipitation with $5 \mu \mathrm{g}$ rabbit antiHistone H3 (acetyl K9) antibody or ChIP rabbit IgG control (both Abcam) at $4^{\circ} \mathrm{C}$. Immunoprecipitated complexes were collected by incubation with recombinant protein $\mathrm{G}$ agarose beads (Roche) for $1-4$ hours at $4^{\circ} \mathrm{C}$. After washing and elution, formaldehyde cross-linking was reversed with a 16 hour overnight incubation at $65^{\circ}$ C. Samples were purified using PCR purification kit columns (Qiagen, Valencia, CA, USA) and used as a template for qPCR.

Enrichment relative to input was calculated from qPCR values from ChIP templates as follows. Standard curves were generated from serial 10-fold dilutions of $10 \%$ of input DNA and plotted on a $\log 10$ scale to obtain the linear relationship between $\mathrm{Ct}$ value and 
Table 2 Primer pairs used for qRT-PCR expression analysis

\begin{tabular}{|c|c|c|}
\hline Gene & $\underline{\text { Forward primer }}$ & Reverse primer \\
\hline Ascl1 & AACCGGGTCAAGTTGGTCAA & CGTCTCCACCTTGCTCATCTTC \\
\hline Cond1 & AGAGGGCTGTCGGCGCAGTA & GGCTGTGGTCTCGGTTGGGC \\
\hline Cdk2 & TGCTGAAATGGTGACCCGCAG & TGCCGAGCCCACTTGGGGAA \\
\hline Cdk4 & GGTGTATGGCGCCGCAGGAA & GCAGGGGATCTTACGCTCGGC \\
\hline p21 (Cdkn1a) & TCCAGGAGGCCCGAGAACGG & CTCCGAACGCGCTCCCAGAC \\
\hline p27 (Cdkn1b) & GCACTGTGGAGCAGACGCCC & TCTGCCAGCAGTCCTGGGGT \\
\hline p57 (Cdkn1c) & CCAATCAGCCAGCCTTCGACCA & CAGGCGCTGCTACGCGCTAT \\
\hline Ctnnb1 & AGGCGAACGGCATTCTGGGC & ACGCACCGTCCTTCGTGCTG \\
\hline Gli1 & GCTTGGATGAAGGACCTTGTG & GCTGATCCAGCCTAAGGTTCTC \\
\hline Hes1 & TGGCGGCTTCCAAGTGGTGC & GATGACCGGGCCGCTGTGAG \\
\hline Hes5 & GCCTGGAGCGGACCAGAGGA & CAGCCGGAGGAGGGAGCCTT \\
\hline c-Myc & ACCCGCTCAACGACAGCAGC & CCGTGGGGAGGACTCGGAGG \\
\hline Neurod1 & CCGCCACACGCCTACA & CAAACTCGGCGGATGG \\
\hline Neurog1 & GACCTGCATCTCTGATCTCG & TGTAGCCTGGCACAGTCCTC \\
\hline Olig2 & CTCCGACGCCAAGTGAGCCG & AATCCCCTAGGCCCAGCCCG \\
\hline Pax6 & AAGCAACAGATGGGCG & GCTTCATCCGAGTCTTC \\
\hline Shh & CACCCCCAATTACAACCCCGACA & TCCAGGCCACTGGTTCATCACAGA \\
\hline Sox1 & CTGGGCGCCCTCGGATCTCT & GGACACGGTGCCCGTGAGTG \\
\hline Sox2 & GAAAGAAAGGAGAGAAGTTGGAG & ATCTGGCGGAGAATAGTTGGG \\
\hline Sox9 & TCGGTGAAGAACGGACAAGC & TGAGATTGCCCAGAGTGCTCG \\
\hline Tcf4 & TGGAGGCCATCCAAGCCCGT & TGCTGGTGGCAACCCTGAACG \\
\hline
\end{tabular}

template concentration. Using this equation, qPCR values were normalized to input and enrichment compared to IgG control samples and differences statistically determined (2-way ANOVA with Bonferroni post-tests). p21 (Cip1/Waf1/Cdkn1a) primers used were GGGT GCAGGGCTGGCTGAAC and TGGACATGGTGC CTGTGGCT, p27 (Kip1/Cdkn1b) primers were CCCAGACCTGCGCGCTACTG and GACCACCGCCTC GCCTCTCT.

\section{Immunofluorescence Cell Fate Quantification}

Passaged adult NSCs were seeded at 2,000 cells/well in ploy-D-lysine coated black frame/white wall 96-well tissue culture plates (Perkin Elmer, Waltham, MA, USA) in NeuroCult ${ }^{\circledR}$ NSC Basal Medium supplemented with mouse NeuroCult ${ }^{\circledR}$ NSC Proliferation Supplement, 20 $\mathrm{ng} / \mathrm{ml} \mathrm{rh}$ EGF, $10 \mathrm{ng} / \mathrm{ml}$ of rh FGF-b, $2 \mathrm{ug} / \mathrm{ml}$ Heparin (all and SAHA or NAB added to culture medium as described previously. In each 96-well plate, SAHA, NaB, DMSO or water vehicle was added to equal numbers of culture wells. After 48 hours medium was replaced with NeuroCult ${ }^{\circledR}$ NSC Basal Medium supplemented with mouse NeuroCult ${ }^{\circledR}$ NSC Differentiation Supplement. Medium was refreshed after 7 days and cells grown for a total of 14 days in culture before fixation in $4 \%$ paraformaldheyde in $1 \times$ PBS. Plates were washed in $1 \times$ PBS and incubated with primary antibody at $4^{\circ} \mathrm{C}$ overnight. Each plate was incubated with a single primary antibody diluted in $1 \times$ PBS containing $1 \%$ NGS, $0.2 \%$ Triton $\mathrm{X}$ -
100. The primary antibodies and dilutions used were as follows: 1: 500 dilution of mouse monoclonal anti-NeuN (Millipore, Billerica, MA, USA); 1:2,000 dilution of mouse monoclonal anti-GFAP (Abcam, Cambridge, MA, USA); 1: 1,000 dilution of rabbit polyclonal anti-Olig2 (Millipore). The following day plates were washed in $1 \times$ PBS and incubated with Alexa Fluor ${ }^{\circledR} 488$ conjugated goat ant-mouse IgG or Alexa Fluor ${ }^{\circledR} 555$ conjugated goat anti-rabbit IgG secondary antibodies (Invitrogen) at 1:1,000 dilution in $1 \times$ PBS for 60 minutes at room temp. Plates were then washed in $1 \times$ PBS and the fluorescence signal in each well measured using a FLUOStar Optima microplate reader (BMG FLUOStar, Cary, NC, USA). The average fluorescence value for each treatment was calculated and data obtained from 6 independent replicates.

\section{Western blot}

Nuclear and cytoplasmic proteins were isolated from adult NSCs using NE-PER ${ }^{\circledR}$ reagents (Thermo Scientific) according to the manufacturer's instructions (Thermo Scientific, Rockford, IL, USA). Protein fractions were stored at $-20^{\circ} \mathrm{C}$ in $1 \mathrm{x}$ Halt ${ }^{\mathrm{TM}}$ Protease Inhibitor Cocktail (Thermo Scientific) until use. Protein extracts were mixed with reducing sample buffer, separated by SDSPAGE and electro-transferred to PVDF membrane. 20 $\mu \mathrm{g}$ protein was loaded per lane. All washes and antisera incubations were performed in 5\% skim milk in TBS with $0.1 \%$ Tween-20. Blots were incubated with primary 
antisera overnight at $4^{\circ} \mathrm{C}$ on a rocking platform. Primary antisera and dilutions used were as follows: 1: 2,000 mouse anti- $\beta$-catenin (BD Biosciences, Franklin Lakes, NJ, USA); 1: 1,000 mouse anti-Gapdh (Millipore): 1,000 mouse anti-Laminin A/C (BD Biosciences). The following day blots were washed 3 times and incubated with a 1:5,000 dilution of peroxidase-conjugated goat antimouse IgG secondary antisera (Cell Signaling Technologies) for 1 hour at room temp. Blots were developed in Immobilon chemiluminescent ECL substrate (Millipore) for 5 minutes at room temp and the fluorescent signal captured using a Fujifilm LAS-3000 phospho-imager (Fujifilm, Valhalla, NY, USA). Immunosignal intensities (protein density) were measured using Fujifilm Multi Gauge software. Images were optimized for brightness and contrast for publication.

\author{
Abbreviations \\ ChIP: chromatin immunoprecipitation; H3: histone 3; H3acK9: acetylated \\ lysine 9 of histone H3; HDAC: histone deacetylase; HDACi: histone \\ deacetylase inhibitor; NaB: sodium butyrate; NSC: neural stem cell; SAHA: \\ suberoylanilide hydroxamic acid
}

\section{Acknowledgements and Funding}

We are grateful to the staff of USU's Biomedical Instrumentation Center for technical assistance. This work is supported by a grant from the Center for Neuroscience and Regenerative Medicine (CNRM).

\section{Author details}

${ }^{1}$ Department of Anatomy, Physiology and Genetics, Center for Neuroscience and Regenerative Medicine, Uniformed Services University of the Health Sciences, 4301 Jones Bridge Road, Bethesda, Maryland 20814, USA. ${ }^{2}$ Department of Biochemistry, Schulich School of Medicine, University of Western Ontario, 112 Siebens-Drake Research Institute, London, Ontario, Canada.

\section{Authors' contributions}

ZQ performed all the cellular and molecular work reported in this manuscript. CD conducted the flow cytometry, associated data analysis and participated in the design of the study. CW participated in the design of the study, in the optimization of chromatin immunoprecipitation protocols and preparation of the manuscript. MD conceived of the study, participated in its design and coordination and drafted the manuscript. All authors have read and approved the final manuscript.

Received: 17 December 2010 Accepted: 26 May 2011

Published: 26 May 2011

\section{References}

1. Lie DC, Colamarino SA, Song HJ, Desire L, Mira H, Consiglio A, Lein ES, Jessberger $S$, Lansford $H$, Dearie AR, et al: Wnt signalling regulates adult hippocampal neurogenesis. Nature 2005, 437(7063):1370-1375.

2. Ahn S, Joyner AL: In vivo analysis of quiescent adult neural stem cells responding to Sonic hedgehog. Nature 2005, 437(7060):894-897.

3. Palma V, Lim DA, Dahmane N, Sanchez P, Brionne TC, Herzberg CD, Gitton Y, Carleton A, Alvarez-Buylla A, Ruiz i Altaba A: Sonic hedgehog controls stem cell behavior in the postnatal and adult brain. Development 2005, 132(2):335-344.

4. Johnston MA, Lim DA: Keeping them quiet: BMPs maintain adult neural stem cell quiescence. Cell Stem Cell 2010, 7(1):9-10.

5. Ables JL, Decarolis NA, Johnson MA, Rivera PD, Gao Z, Cooper DC, Radtke F, Hsieh J, Eisch AJ: Notch1 is required for maintenance of the reservoir of adult hippocampal stem cells. J Neurosci 2010, 30(31):10484-10492.

6. Chapouton P, Skupien P, Hesl B, Coolen M, Moore JC, Madelaine R, Kremmer E, Faus-Kessler T, Blader P, Lawson ND, et al: Notch activity levels control the balance between quiescence and recruitment of adult neural stem cells. J Neurosci 2010, 30(23):7961-7974.

7. Lim DA, Huang YC, Swigut T, Mirick AL, Garcia-Verdugo JM, Wysocka J, Ernst P, Alvarez-Buylla A: Chromatin remodelling factor Mll1 is essential for neurogenesis from postnatal neural stem cells. Nature 2009, 458(7237):529-533.

8. Jawerka M, Colak D, Dimou L, Spiller C, Lagger S, Montgomery RL, Olson EN, Wurst W, Gottlicher M, Gotz M: The specific role of histone deacetylase 2 in adult neurogenesis. Neuron Glia Biol 2010, 1-15.

9. Ma DK, Marchetto MC, Guo JU, Ming GL, Gage FH, Song H: Epigenetic choreographers of neurogenesis in the adult mammalian brain. Nat Neurosci 2010, 13(11):1338-1344.

10. Buchwald M, Kramer OH, Heinzel T: HDACi-targets beyond chromatin. Cancer Lett 2009, 280(2):160-167.

11. Kazantsev AG, Thompson LM: Therapeutic application of histone deacetylase inhibitors for central nervous system disorders. Nat Rev Drug Discov 2008, 7(10):854-868.

12. Kim HJ, Leeds $P$, Chuang DM: The HDAC inhibitor, sodium butyrate, stimulates neurogenesis in the ischemic brain. J Neurochem 2009, 110(4):1226-1240.

13. Kim HJ, Rowe M, Ren M, Hong JS, Chen PS, Chuang DM: Histone deacetylase inhibitors exhibit anti-inflammatory and neuroprotective effects in a rat permanent ischemic model of stroke: multiple mechanisms of action. J Pharmacol Exp Ther 2007, 321(3):892-901.

14. Fischer A, Sananbenesi F, Wang X, Dobbin M, Tsai LH: Recovery of learning and memory is associated with chromatin remodelling. Nature 2007, 447(7141):178-182.

15. Dash PK, Orsi SA, Moore AN: Histone deactylase inhibition combined with behavioral therapy enhances learning and memory following traumatic brain injury. Neuroscience 2009, 163(1):1-8.

16. Vecsey CG, Hawk JD, Lattal KM, Stein JM, Fabian SA, Attner MA, Cabrera SM, McDonough CB, Brindle PK, Abel T, et al: Histone deacetylase inhibitors enhance memory and synaptic plasticity via CREB:CBP-dependent transcriptional activation. J Neurosci 2007, 27(23):6128-6140.

17. Siebzehnrubl FA, Buslei R, Eyupoglu IY, Seufert S, Hahnen E, Blumcke I: Histone deacetylase inhibitors increase neuronal differentiation in adult forebrain precursor cells. Exp Brain Res 2007, 176(4):672-678.

18. Schroeder FA, Lin CL, Crusio WE, Akbarian S: Antidepressant-like effects of the histone deacetylase inhibitor, sodium butyrate, in the mouse. Biol Psychiatry 2007, 62(1):55-64.

19. Uo T, Veenstra TD, Morrison RS: Histone deacetylase inhibitors prevent p53-dependent and p53-independent Bax-mediated neuronal apoptosis through two distinct mechanisms. J Neurosci 2009, 29(9):2824-2832.

20. Salminen A, Tapiola T, Korhonen P, Suuronen T: Neuronal apoptosis induced by histone deacetylase inhibitors. Brain Res Mol Brain Res 1998, 61(1-2):203-206.

21. Wang $Y$, Wang $X$, Liu L: HDAC inhibitor trichostatin A-inhibited survival of dopaminergic neuronal cells. Neurosci Lett 2009, 467(3):212-216.

22. Kelly WK, O'Connor OA, Krug LM, Chiao JH, Heaney M, Curley T, MacGregore-Cortelli B, Tong W, Secrist JP, Schwartz L, et al: Phase I study of an oral histone deacetylase inhibitor, suberoylanilide hydroxamic acid, in patients with advanced cancer. J Clin Oncol 2005, 23(17):3923-3931.

23. Stimson L, La Thangue NB: Biomarkers for predicting clinical responses to HDAC inhibitors. Cancer Lett 2009, 280(2):177-183.

24. Wilting $\mathrm{RH}$, Yanover $\mathrm{E}$, Heideman $\mathrm{MR}$, Jacobs $\mathrm{H}$, Horner J, van der Torre J, DePinho RA, Dannenberg JH: Overlapping functions of Hdac1 and Hdac2 in cell cycle regulation and haematopoiesis. Embo J 2010, 29(15):2586-2597.

25. Yamaguchi T, Cubizolles F, Zhang Y, Reichert N, Kohler H, Seiser C, Matthias P: Histone deacetylases 1 and 2 act in concert to promote the G1-to-S progression. Genes Dev 2010, 24(5):455-469.

26. Zupkovitz G, Grausenburger R, Brunmeir R, Senese S, Tischler J, Jurkin J, Rembold M, Meunier D, Egger G, Lagger S, et al: The cyclindependent kinase inhibitor p21 is a crucial target for histone deacetylase 1 as a regulator of cellular proliferation. Mol Cell Biol 2010, 30(5):1171-1181.

27. Lagger G, O'Carroll D, Rembold M, Khier H, Tischler J, Weitzer G, Schuettengruber B, Hauser C, Brunmeir R, Jenuwein T, et al: Essential function of histone deacetylase 1 in proliferation control and CDK inhibitor repression. Embo J 2002, 21(11):2672-2681. 
28. Marks PA, Xu WS: Histone deacetylase inhibitors: Potential in cancer therapy. J Cell Biochem 2009, 107(4):600-608.

29. Di Bernardo G, Squillaro T, Dell'Aversana C, Miceli M, Cipollaro M, Cascino A, Altucci L, Galderisi U: Histone deacetylase inhibitors promote apoptosis and senescence in human mesenchymal stem cells. Stem Cells Dev 2009, 18(4):573-581.

30. Hsieh J, Nakashima K, Kuwabara T, Mejia E, Gage FH: Histone deacetylase inhibition-mediated neuronal differentiation of multipotent adult neural progenitor cells. Proc Natl Acad Sci USA 2004, 101(47):16659-16664.

31. Ye F, Chen Y, Hoang T, Montgomery RL, Zhao XH, Bu H, Hu T, Taketo MM, van $\mathrm{Es} \mathrm{JH}$, Clevers $\mathrm{H}$, et al: HDAC1 and HDAC2 regulate oligodendrocyte differentiation by disrupting the beta-catenin-TCF interaction. Nat Neurosci 2009, 12(7):829-838.

32. Chen JS, Faller DV: Histone deacetylase inhibition-mediated posttranslational elevation of p27KIP1 protein levels is required for G1 arrest in fibroblasts. J Cell Physiol 2005, 202(1):87-99.

33. Mathew OP, Ranganna K, Yatsu FM: Butyrate, an HDAC inhibitor, stimulates interplay between different posttranslational modifications of histone $\mathrm{H} 3$ and differently alters $\mathrm{G} 1$-specific cell cycle proteins in vascular smooth muscle cells. Biomed Pharmacother 2010.

34. Ocker M, Schneider-Stock R: Histone deacetylase inhibitors: signalling towards p21cip1/waf1. Int J Biochem Cell Biol 2007, 39(7-8):1367-1374.

35. Vigushin DM, Coombes RC: Histone deacetylase inhibitors in cancer treatment. Anticancer Drugs 2002, 13(1):1-13.

36. Lai K, Kaspar BK, Gage FH, Schaffer DV: Sonic hedgehog regulates adult neural progenitor proliferation in vitro and in vivo. Nat Neurosci 2003, 6(1):21-27.

37. Androutsellis-Theotokis A, Leker RR, Soldner F, Hoeppner DJ, Ravin R, Poser SW, Rueger MA, Bae SK, Kittappa R, McKay RD: Notch signalling regulates stem cell numbers in vitro and in vivo. Nature 2006, 442(7104):823-826

38. Elmi M, Matsumoto Y, Zeng ZJ, Lakshminarasimhan P, Yang W, Uemura A, Nishikawa S, Moshiri A, Tajima N, Agren H, et al: TLX activates MASH1 for induction of neuronal lineage commitment of adult hippocampal neuroprogenitors. Mol Cell Neurosci 2010, 45(2):121-131.

39. Marks PA, Jiang $X$ : Histone deacetylase inhibitors in programmed cell death and cancer therapy. Cell Cycle 2005, 4(4):549-551.

40. Huangfu D, Maehr R, Guo W, Eijkelenboom A, Snitow M, Chen AE, Melton DA: Induction of pluripotent stem cells by defined factors is greatly improved by small-molecule compounds. Nat Biotechnol 2008, 26(7):795-797.

41. Liang G, Taranova O, Xia K, Zhang Y: Butyrate promotes induced pluripotent stem cell generation. J Biol Chem 2010, 285(33):25516-25521.

42. Ruau D, Ensenat-Waser R, Dinger TC, Vallabhapurapu DS, Rolletschek A, Hacker C, Hieronymus T, Wobus AM, Muller AM, Zenke M: Pluripotency associated genes are reactivated by chromatin-modifying agents in neurosphere cells. Stem Cells 2008, 26(4):920-926.

43. Mitsui K, Tokuzawa Y, Itoh H, Segawa K, Murakami M, Takahashi K, Maruyama M, Maeda M, Yamanaka S: The homeoprotein Nanog is required for maintenance of pluripotency in mouse epiblast and ES cells. Cell 2003, 113(5):631-642.

44. Baltus GA, Kowalski MP, Tutter AV, Kadam S: A positive regulatory role for the mSin3A-HDAC complex in pluripotency through Nanog and Sox2.J Biol Chem 2009, 284(11):6998-7006.

45. Li Y, Zhang X, Polakiewicz RD, Yao TP, Comb MJ: HDAC6 is required for epidermal growth factor-induced beta-catenin nuclear localization. $J$ Biol Chem 2008, 283(19):12686-12690.

46. Wexler EM, Geschwind DH, Palmer TD: Lithium regulates adult hippocampal progenitor development through canonical Wht pathway activation. Mol Psychiatry 2008, 13(3):285-292.

47. Riester D, Hildmann C, Schwienhorst A: Histone deacetylase inhibitorsturning epigenic mechanisms of gene regulation into tools of therapeutic intervention in malignant and other diseases. Appl Microbiol Biotechnol 2007, 75(3):499-514.

doi:10.1186/1471-2202-12-50

Cite this article as: Zhou et al:: Histone deacetylase inhibitors SAHA and sodium butyrate block G1-to-S cell cycle progression in neurosphere formation by adult subventricular cells. BMC Neuroscience 2011 12:50.

\section{Submit your next manuscript to BioMed Central and take full advantage of:}

- Convenient online submission

- Thorough peer review

- No space constraints or color figure charges

- Immediate publication on acceptance

- Inclusion in PubMed, CAS, Scopus and Google Scholar

- Research which is freely available for redistribution

Submit your manuscript at www.biomedcentral.com/submit 